\title{
Agentes comunitarios de salud, medicalización y acción pastoral del Estado
}

\section{Community health agents, medicalization and pastoral action of the State}

\author{
Ángel Estigarribia ${ }^{a}$
}

\begin{abstract}
Resumen
Las políticas públicas van más allá del servicio social específico, la utilidad de las mismas, para el aparato estatal es amplia. El Estado tiene como primer objetivo fortalecer su poder. La salud pública es un aspecto central de la intervención política y dentro de ella, la estrategia de Atención Primaria de la Salud (APS) cumple un papel relevante.

Dentro de la estrategia de APS los Agentes Comunitarios de Salud (ACS), son la puerta de entrada del sistema de salud y también el mecanismo más amigable de investigación estatal. Desarrollan una tarea fundamental: son el puntal indispensable para la adherencia de los pacientes al tratamiento médico y construyen el marco de confianza para el proceso de búsqueda, recolección y procesamiento de los datos sobre los individuos y el entorno, para el acceso del Estado a la intimidad de las personas y finalmente para el control social.
\end{abstract}

Palabras clave: salud pública, agentes comunitarios de salud, control social.

\begin{abstract}
Public policies go beyond the specific social service, the utility of the same, for the state apparatus is broad. The first objective of the State is to strengthen its power. Public health is a central aspect of political intervention and within it, the strategy of Primary Health Care (APS) plays a relevant role.

Within the APS strategy, the Community Health Agents (ACS) are the gateway to the health system and also the most friendly state research mechanism. They develop a fundamental task: they are the essential prong for the adherence of patients to medical treatment and build the framework of trust for the process of searching, collecting and processing data on individuals and the environment, for State access to the Intimacy of people and finally for social control.
\end{abstract}

Keywords: public health, community health agents, social control.
Kera Yvoty: reflexiones sobre la cuestión social. Vol. 2, 2017, $67-83$.

ISSN (impreso): 2519-7797

a Centro de Investigaciones en Filosofía y Ciencias Humanas (CIF), Paraguay.

Correspondencia a: aestigarribia@yahoo.com

Cita:

Estigarribia, A. (2017).

Agentes comunitarios de salud, medicalización y acción pastoral del Estado. Kera Yvoty: reflexiones sobre la cuestión social, 2, 83-99.

Recibido:

10 agosto 2017

Aceptado:

26 noviembre 2017 


\section{Introducción}

El presente artículo es parte de una investigación más amplia sobre la medicalización de la vida de las personas por parte del Estado, en este caso específico se propone examinar el rol que cumplen los Agentes Comunitarios de Salud (ACS) dentro de la estrategia de Atención Primaria de Salud y en el sistema de control social del Estado. El análisis explora, la relación entre los ACS y la población, la interacción entre las organizaciones sociales y los servicios de salud, el papel de las ACS en la gestión de la información sobre las personas y comunidades.

El objetivo del estudio es ejemplificar que toda política pública tiene como primera misión fortalecer el poder del Estado y conlleva procedimientos que sirven para la consolidación del poder y que operan con independencia de los objetivos específicos de los programas de gobierno.

Cuanto más humanitarios aparecen las estrategias y los programas, cuanto más amigables sean los métodos, más profundos son los lazos que el Estado establece con la población y más eficaces se vuelven los sistemas de control. Los ACS son los nervios del sistema que enlazan a las comunidades con el centro estatal.

\section{Estado y medicalización}

La medicina moderna es social, políticamente administrada. El Estado en la actualidad toma en sus manos la salud de la gente, ésta dejó de ser un asunto privado. La vida saludable, más que un problema personal es un objetivo político; es deber del Estado velar por ella. La población saludable y laboriosa, la alta tasa de natalidad y la baja mortalidad maternoinfantil para la reproducción de la fuerza de trabajo son las prioridades a cuyo servicio debe estar el sistema sanitario.

Si la pobreza, el hacinamiento, la nutrición insuficiente, son las fuentes de morbilidad y mortalidad más frecuentes, también lo son de los disturbios y protestas sociales. Virchow llegó a afirmar que la política no es más que medicina en una escala más amplia'. Se trata de enfrentar los problemas sociales más que las patologías de los individuos y de establecer sistemas de diagnósticos y vigilancias de la población que hagan posibles soluciones a gran escala (Waitzkin, 2006).

La salud depende menos de la atención médica que de las condiciones de vida de la población. "Virchow atribuía el brote [epidémico] a un conjunto de factores sociales y económicos y, en consecuencia, esperaba poco de cualquier tratamiento médico" (Rosen, 2005, p. 78). El problema sanitario estaba en los determinantes sociales y, consecuentemente, se debía buscar el origen de las patologías en las relaciones sociales. Las causas de la incidencia de la morbilidad de la población son distintas de las causas de la incidencia de los problemas de salud individuales (Mackenbach, 2009). Tanto el diagnóstico como la solución dependían menos de la técnica médica que del tratamiento de la cuestión social.

La búsqueda de las causas sociales de las epidemias deriva en la investigación de todos los aspectos que puedan afectar la estabilidad social. La gestión del cuerpo, la administración de las poblaciones y el abordaje de los determinantes sociales, son ahora principios políticos de primer orden para la salud pública (Davis \& Chapa, 2015) y para la estabilidad del sistema (Oleskin, 2012).

En el siglo XX, cobra importancia internacional la gestión estatal de la salud. Desde los años 1940-50 "el cuerpo del individuo se convierte en uno de los objetivos principales de la intervención del Estado, uno de los grandes objetos de los que el propio Estado debe hacerse cargo" (Foucault, 1978, p. 19). A partir de la Conferencia de Alma Ata de 1978, la

\footnotetext{
1 "Die Medizin ist eine soziale Wissenschaft, und die Politik ist nichts weiter als Medizin im Großen" (Segura del Pozo, 2009).
} 
Organización Mundial de la Salud (OMS) dejó en claro que el Estado debe asumir la salud de la población y además dejó nítidamente establecida la estrategia:

Los gobiernos tienen la obligación de cuidar la salud de sus pueblos, obligación que sólo puede cumplirse mediante la adopción de medidas sanitarias y sociales adecuadas. Uno de los principales objetivos sociales de los gobiernos, de las organizaciones internacionales y de la comunidad mundial entera en el curso de los próximos decenios debe ser el de que todos los pueblos del mundo alcancen en el año 2000 un nivel de salud que les permita llevar una vida social y económicamente productiva. La atención primaria de salud es la clave para alcanzar esa meta como parte del desarrollo conforme al espíritu de la justicia social (PAHO, 1978, p. falta).

Con la estrategia de APS, el Estado adopta como obligación la salud de la población y se arroga el derecho a adoptar las medidas políticas que considere pertinentes para el efecto.

\section{Política de rebaños}

La desatención del cuerpo, las inadecuadas condiciones de vida y de trabajo conllevan morbilidad extrema, baja tasa de natalidad y altos índices de mortalidad. Los empleadores necesitan disponer de una fuerza laboral abundante y sana además de seguras condiciones de reproducción de la mano de obra.

Aunque persiste la estratificación social en la atención sanitaria, desde el Estado la prioridad política es la medicina social y universal. El Estado necesita vigilar a todos y especialmente a aquellos sectores potencialmente desestabilizadores.

La autoridad pública define la conducta saludable y determina los procedimientos para lograrla. En nombre de la ciencia, el poder político se encamina a la administración de la vida. No es suficiente luchar contra la enfermedad, hay que acometer la gestión corporal

"Hoy la medicina está dotada de un poder autoritario con funciones normalizadoras que van más allá de la existencia de enfermedades y la demanda del enfermo" (Foucault, 1978, p. 26). La relación medicina-poder político ha establecido que la vida saludable depende de varios factores que contribuyen a crear mejores o peores condiciones de vida y de trabajo.

Los determinantes sociales de la salud son las circunstancias en que las personas nacen, crecen, viven, trabajan y envejecen, incluido el sistema de salud. Esas circunstancias son el resultado de la distribución del dinero, el poder y los recursos a nivel mundial, nacional y local, que depende a su vez de las políticas adoptadas. (OMS, 2012, p. falta)

Un diagnóstico social, munido de un sistema de detección y vigilancia dinámico, es la condición para el establecimiento de estrategias estatales sanitarias. En este marco la OMS exhorta a las naciones miembros a: que haga de los determinantes sociales de la salud un principio rector para la aplicación de medidas, entre ellas indicadores objetivos para seguir los determinantes sociales de la salud, en todas las áreas de trabajo pertinentes y fomente la atención a dichos determinantes con miras a reducir las inequidades sanitarias como objetivo de todas las áreas de trabajo de la Organización, y en particular de los programas de salud pública prioritarios. (OMS, 2009, p. 4)

Al tomar los determinantes sociales como uno de los campos de la ciencia médica, ésta se vuelve una disciplina eminentemente política y las consecuencias de este paso son múltiples y van más allá del campo de la salud. Un efecto inmediato es que no importa 
la voluntad del individuo. "La medicina se dedica a otros campos que no son las enfermedades, y con arreglo a un sistema de relaciones no regido por la demanda del paciente" (Foucault, 1978, p. 27).

El Estado asume el deber de la atención integral de la población, su obligación es cuidar del "rebaño" y toma medidas más allá de la voluntad de los individuos. La vigilancia permanente en nombre de esta cobertura se vuelve una necesidad científica y política. La salud de la población es un discurso que permite al Estado adoptar estrategias de control para garantizar su cometido.

Aquí tiene lugar aquella observación profunda que hacía Foucault en el desarrollo de su teoría pastoral de la gubernamentalidad. El pastorado es un arte político, en principio con características religiosas, sin embargo, en occidente fue adoptado como método en diferentes ámbitos de poder.

En el cristianismo el pastorado produjo todo un arte de conducir, dirigir, guiar, llevar de la mano, manipular a los hombres, un arte de seguirlos y moverlos paso a paso, un arte cuya función es tomarlos a cargo colectiva e individualmente a lo largo de toda su vida y en cada momento de su existencia. (Foucault, 2006, p. 192)

El pastor, para el ejercicio de su método de conducción debe conocer a todos y cada uno, profundamente, íntimamente. Es lo que caracteriza de manera original a esta forma de gobierno. Para el poder pastoral es muy importante el manejo de la información de todos y cada uno de los miembros del rebaño. Para eso es menester adoptar instrumentos que hagan posible la observación meticulosa. También es importante el acceso a la profundidad del individuo, a lo más recóndito, al pensamiento, a los miedos, a las ilusiones, a través del método de la confesión. El pastor para guiar necesita conocer a su rebaño, adelantarse, prever (Foucault, 2006, p. 155). Para eso es el oído y la palabra, la inquisición, el cuestionario, la declaración. Para el Estado indispensable que nadie quede fuera de los registros, sanos y enfermos.

En el

gubernamentalización, el Estado coopta y convierte a los individuos en agentes para el examen de los riesgos y para el monitoreo de las personas vulnerables. La gestión pastoral se ejerce a través de miles de ojos y oídos del pastor presentes en la comunidad. En la campaña contra las epidemias como el dengue, chikungunya y zika el eje sobre el que ha girado el sistema de prevención ha sido el involucramiento de la población en la detección y eliminación de los criaderos de mosquitos $^{2}$. El problema de la epidemia se convertía en un asunto de responsabilidad social, en un acontecimiento colectivo de higiene y, sobre todo, en un plan de investigación de los posibles focos.

"Las acciones institucionales generadas con la EGI Dengue buscan fomentar el cambio de conducta tanto individual como colectiva para un mejor ordenamiento ambiental en función de la prevención del Dengue" (Paraguay, Ministerio de Salud Pública y Bienestar Social (sf). Todos controlan a todos, el derecho a la salud conlleva deberes y exige mirar minuciosamente los detalles de la vivienda propia y ajena a fin de detectar los criaderos de vectores. Este ejemplo ilustra el tipo de orientación estatal para producir nuevos comportamientos.

2 El Dengue es un problema de salud pública con múltiples determinantes, que van desde microfactores -como el mecanismo de transmisión propio de la enfermedad o las particularidades de salud de cada persona afectada- hasta macrofactores políticos, ambientales y sociales. En Paraguay, los factores ambientales y sociales actúan con mucha fuerza sobre el problema del Dengue. Entendiendo que éste rebasa las fronteras del Sector Salud, el MSP y BS sienta a la articulación de acciones interinstitucionales e intrasectoriales entre las prioridades de su EGI y apela a las comunidades como agentes fundamentales de cambio. (Paraguay, Ministerio de Salud Pública y Bienestar Social, sf) 
Irrumpe así un tipo de compromiso social asociado a la observación de las condiciones de vida del vecindario. El manejo pastoral de la salud pública significa que no puede haber nada fuera del cuidado del "pastor". Él es el que elige a su rebaño, y se obliga a cuidar de todos (Foucault, 2006). Nadie puede evitar las acciones sanitarias del poder político, nadie puede escapar a la vigilancia salubrista. No es una cuestión de elección, no es una atención por demanda. No son sólo los funcionarios quienes controlan, el brazo político se ha extendido hasta la comunidad misma, el papel de los Agentes Comunitarios de Salud (ACS) se torna estratégico para la incorporación de la población en la tarea del Estado. Además de los diversos registros institucionales e instrumentos de recolección de datos, está la visión, la presencia y la acción de la comunidad, de los grupos, de los vecinos. Mirada inquisidora y humanista. Controlar para prevenir y ayudar.

El pastorado funciona como una red, con raicillas interminables, como un rizoma (Deleuze \& Guattari, 1994). El Estado desde la salud pública involucra políticamente a la población en su gestión, produce un funcionariado ad honorem (Bourdieu, 1998).

Más que a los enfermos, hay que vigilar a los sanos. Del manejo de eventos patológicos a la prevención de sucesos. "La salud pública y la epidemiología delimitaron el concepto de riesgo y de prevención, introduciendo la enfermedad -aunque sólo sea como probabilidad- en el mundo de los sanos" (Martínez, sf, p. 4). El individuo sano debe ser examinado porque es un agente patológico en potencia.

El Estado busca adelantarse a las situaciones, procura la separación oportuna de los agentes morbosos, para ello es imprescindible la presencia permanente de un sistema de monitoreo sanitario y la agitación del peligro latente. Lo que motiva la acción pública es la amenaza patológica, la acción se dirige a las poblaciones, al territorio social 3 . El trabajo de inteligencia es fundamental para el enfoque promocional y para que la población se involucre en la gestión. Esto implica la acción participativa en la identificación de los problemas que pueden generar procesos negativos, desde problemas medioambientales, focos epidémicos, modos de vida inadecuados.

"La política de promoción de la salud requiere que se identifiquen y eliminen los obstáculos que impidan la adopción de medidas políticas que favorezcan la salud en aquellos sectores no directamente implicados en la misma” (PAHO, 1986). La autoridad pública arbitra los medios para que la población, en el proceso de cuidado de su salud, desarrolle mecanismos de control social.

La noción de control social significa "procesos de desarrollo de la conformidad" (Janowitz, 1975, p. 11). Apunta a la consecución de compromisos sociales para la reducción de la coerción y la eliminación de la miseria humana (Janowitz, 1975), objetivos humanistas que conlleva la paz social, incluye el compromiso con procedimientos para redefinir metas sociales a fin de afirmar el papel de la racionalidad (Janowitz, 1975). La participación social es indispensable y empieza con la aceptación de los métodos institucionales y la solidaridad con ellos (empoderamiento). El objetivo inmediato es una población con valores, hábitos y costumbres internalizados sin coacción directa conforme al ordenamiento social dominante.

Cuando el sistema de control es asumido por la comunidad es cuando ejerce todo su poderío. Cuanto menos represiva aparezca una iniciativa estatal, más fácilmente puede ser asimilada. La

\footnotetext{
3 Llamamos territorio social al lugar donde vive y con el que se identifica la población, donde se dan o no las condiciones para una vida digna. Espacio vivo, dinámico, cambiante que se construye y reconstruye permanentemente y donde se producen y reproducen los determinantes y exposiciones sociales a factores estructurales (Paraguay. Ministerio de Salud Pública y Bienestar Social, 2012e, p. 9).
} 
asunción de los diversos sistemas de vigilancia, registro y comunicación, es el consenso arbitrado desde el poder, cuando el sector dominante logra que la población colabore, participey, sobre todo, que amplíe los efectos del sistema.

La aparente insignificancia de una hoja de encuesta, o de una entrevista de un trabajador social, o de una inocente pregunta del médico, o de un rutinario registro de una enfermera, o de un burocrático expediente clínico, indica la enorme complejidad del sistema político, cuyo funcionamiento da sentido al aparente acto dislocado, al hecho insignificante. "Cuando la maquinaria deviene planetaria o cósmica, los agenciamientos tienden cada vez más a miniaturizarse o a devenir microagenciamientos" (Deleuze \& Guattari, 1994, p. 220).

El Estado no hace nada sin que organice y dirija sus acciones hacia lo más importante: el agenciamiento de los individuos para que actúen, ellos mismos, como agentes de control comunitario. La adhesión al tratamiento clínico, el ejercicio de la promoción de la salud, la afiliación a los grupos de gestión participativa de algún segmento de los servicios estatales, son mecanismos sencillos, inocentes y eficaces que hacen al funcionamiento del dispositivo universal de investigación y seguimiento.

El Agente Comunitario de Salud en la estrategia de APS hace una labor de investigación y, a la vez ejerce de trabajador social, árbitro de los conflictos familiares y comunitarios, protector de la salud, pero, sobre todo, gestor de la adhesión a las políticas públicas.

\section{El agente comunitario de salud y su equipo}

El Equipo de Salud de la Familia (ESF) es el cuerpo de funcionarios de una Unidad de la Salud de la Familia, la célula básica de la estrategia de APS. La primera tarea del ESF es la presentación a la comunidad lo que establece un acontecimiento importante. En la mayoría de estos lugares es la primera vez que tienen un equipo de salud y, particularmente, un médico. La segunda actividad es la realización de un censo de la población asignada y el georreferenciamiento de la vivienda familiar en un mapa.

El ACS tiene como tarea principal la realización de la territorialización y mapeo del área de actuación del equipo, la identificación de grupos, familias e individuos expuestos a riesgos, incluyendo aquellos que son ocupacionales y laborales. La tarea es continua porque corresponde la actualización permanente de dichos informes.

Los ESF tienen que asumir el cuidado de la salud de la población adscrita en el ámbito de las Unidades de Salud de la Familia, ya sea en el domicilio como en los espacios comunitarios (escuelas, asociaciones, clubes, etc.). Deben garantizar la integralidad de la atención mediante la promoción de la salud que incluye actividades preventivas, educativas, curativas, de rehabilitación y de vigilancia de la salud. Deben prestar la atención de las demandas espontáneas, las consultas programadas y las urgencias, el desarrollo de las acciones programáticas en el servicio y en la comunidad, la búsqueda activa de enfermedades de notificación obligatoria $\mathrm{u}$ otras situaciones de riesgo para la salud colectiva e informar a las instancias correspondientes. Deben escuchar y atender las necesidades de los usuarios en todas las acciones, proporcionando atención humanizada, estableciendo o reforzando el vínculo del ESF con las personas. Responsabilizarse de la población adscrita, manteniendo la coordinación del cuidado tanto a nivel de las USF, como con las otras redes del sistema cuando sea necesario. Planificar y evaluar las acciones del equipo, a partir del análisis de datos disponibles.

Desde la instalación de las USF "se cuenta con información más detallada de la población y de las atenciones realizadas, 
esto permite la recolección de datos relevantes y actualizados, y favorece la toma de decisiones oportunas" (Caballero, Denis, Flecha et alt. 2010, p. 45). La recolección de estas informaciones relevantes permite identificar líderes, organizaciones $\mathrm{u}$ otros recursos de la comunidad que puedan potenciar las acciones con el equipo. La gestión de la información con la propia población garantiza la calidad del registro de las actividades en el Sistema Nacional de Información.

Los ESF deben promover la movilización y participación de la comunidad en la gestión social del territorio. Los ESF deben participar de las actividades de educación permanente diseñados para sus integrantes. Realizar reuniones periódicas para organizar y planificar el trabajo grupal, programar y evaluar las acciones al interior de la USF y con la comunidad. (Ministerio de Hacienda, 2011, p. 30).

El hecho de saber escuchar los problemas de la gente implica la posibilidad del acceso a un mundo de informaciones sobre sus preocupaciones y problemas. Se genera una relación de confianza que permitirá en el futuro la adherencia al tratamiento y al proceso interrogatorio, fundamentales para el cuidado de la salud y para la actualización de la base de datos de las personas, de las familias y del entorno inmediato.

El conocimiento cotidiano y la confianza de la población adscripta ayudaránalaubicación delasorganizaciones existentes, a la identificación de los líderes, de sus actividades y de sus potencialidades, en principio para los objetivos de la salud pública y, en general para los fines del Estado.

La acción de vigilancia vecinal opera sobre algunos indicadores trazadores: las familias que no vacunan a sus hijos, sobre la violencia intrafamiliar, sobre los adictos, sobre los ancianos sin cuidado, sobre las adolescentes embarazadas, sobre los agentes comunitarios de salud, sobre las enfermeras y médicos de las Unidades de Salud de la Familia. Se controlan las visitas de los agentes de salud, se observan las reuniones de los clubes de hipertensos y diabéticos, de los grupos de violencia intrafamiliar, se registran las reuniones de los grupos que buscan reducir los daños de las adicciones.

Ninguna institución del Estado puede lograr tanta colaboración de la comunidad, de las organizaciones sociales, de los líderes e individuos como el MSPyBS.

\section{El poder amigable}

El rol del agente comunitario de salud (ACS) es uno de los aspectos más destacados por las primeras evaluaciones oficiales de la estrategia de APS: "Las USF incorporan prácticas nuevas, como el censo comunitario y las visitas comunitarias, para conocer el perfil de la población asignada y la interacción de la misma con el ESF" (Caballero, Denis, Flecha et al., 2010, p. 5).

Los ACS no son simplemente la polea de transmisión de las políticas de salud al ámbito de los hogares, sino que son fundamentales para la «interacción» entre las familias y las USF. Por medio de estos agentes, la vigilancia de la salud y de las condiciones de vida, se realiza con independencia de la acción y demanda de los pacientes. "El principal escenario de trabajo de los ACS es la comunidad: las casas de las familias, las escuelas, los lugares de trabajo, los lugares de culto, los espacios de encuentro, de esparcimiento y en las calles" (Paraguay. Ministerio de Salud Pública y Bienestar Social, 2012c, p. 17).

Los ACS son los principales gestores de la confianza, obtienen información íntima que difícilmente otros agentes lo consiguen. Tienen la capacidad de completar, corregir y mejorar los datos del censo, hacen que este sea el único actualizado y dinámico en el país.

Los territorios sociales son organizados en "micro territorios" en función de la acción de los ACS. Estos tienen a su cargo dichos ámbitos, son 
responsables de sus habitantes, los tienen que conocer y, de hecho, los conocen por nombre y apellido e incluso por sus apodos. "su trabajo ayuda a que se conozcan situaciones problemáticas que afectan la calidad de vida de las familias, situaciones individuales y colectivas que ponen en peligro la salud o la vida de las personas" (Paraguay. Ministerio de Salud Pública y Bienestar Social, 2012c, p. 20).

Conocen sus actividades, sus grupos de amigos, sus aficiones, son testigos de sus presencias y ausencias. Saben en qué trabajan, donde pasan el tiempo, conocen sus proyectos y hasta sus expectativas. Y lo que no saben pueden preguntar, ya que por la confianza lograda obtendrán respuesta. Todo lo que el Estado considera «situaciones problemáticas», que afectan la vida de las personas podrán ser conocidas, monitoreadas e informadas a través de los ACS. Podrán obtenerse aquellas informaciones que pueden ser de utilidad tanto para la salud, como para los diversos perfiles de las distintas dependencias ministeriales "...las condiciones de vivienda, de ocupación, ingresos, hábitos, creencias, valores, religión, opciones de ocio, etc." (Paraguay. Ministerio de Salud Pública y Bienestar Social, 2012d, p. 13). Los ACS son los más importantes gestores de información confidencial del Estado, instalados a escasos metros de las viviendas, en relación amigable y permanente con los habitantes de los barrios.

Los agentes tienen una importancia clave en la organización de las actividades de los individuos y los grupos. Hacen de gerentes de las acciones comunitarias, lideran el proceso organizativo. La verdadera puerta de entrada al sistema de salud es el agente comunitario. El evento sanitario más regular es la visita mensual a las familias ${ }^{4}$.

4 La visita casa por casa es una de las actividades más importantes de/la ACS en la comunidad, de las más delicadas y por ello, una de las mejor planificadas, al punto que el MSPBS ha producido una "Guía de
La presencia de agentes comunitarios permite llegar de mejor manera a la población adscripta y logra una participación más activa de las actividades de los ESF, además se logra un mejor control del cuidado de la salud de la población, las visitas mensuales a todas las familias, logra reunir en actividades comunitarias, mingas ambientales, formación de clubes por patologías, y grupo etario con el fin de lograr la prevención y promoción de la salud. (Paraguay. Ministerio de Hacienda, 2011, p. 11).

Lo más importante, sin embargo, es la relación misma. "cuando los/as ACS establecen vínculos con las familias se instala una relación muy fuerte donde se entrecruzan afectos, responsabilidades y compromisos" (Paraguay. Ministerio de Salud Pública y Bienestar Social, 2012c, p. 33). Esta relación íntima es un estimulante muy importante para el acceso a la información confidencial de las personas, para el cuestionario recurrente, para la actualización de los datos. El hecho de ser un agente que vela por la salud de la población es el mejor salvoconducto que un funcionario del Estado puede tener, para hurgar en la vida de los individuos y las familias5. Por ello la visita domiciliaria es la clave para la estrategia de la APS. Si las USF son la puerta de entrada al sistema, el agente comunitario es el verdadero pasaporte y la visita domiciliaria la llave. Por algo esta visita es lo principal en los ACS .

visitas domiciliarias" (Paraguay. Ministerio de Salud Pública y Bienestar Social, 2012d).

5 Por regla general, las visitas son muy apreciadas y los pobladores reciben bien a los miembros del equipo. Vale la pena recordar que nadie está obligado a abrir su casa a nadie (Paraguay. Ministerio de Salud Pública y Bienestar Social, 2012d, p. 10).

6 La visita domiciliaria es la principal actividad de los Agentes Comunitarios de Salud (ACS). La construcción de un sólido y duradero vínculo con las familias es necesaria para el desarrollo de las acciones de promoción. (Paraguay. Ministerio de Salud Pública y Bienestar Social, 2012d, p.10). 
El rol vigilante de los ESF radica, de modo particular, en los agentes comunitarios. Actualizan las informaciones epidemiológicas, tanto de las enfermedades transmisibles como de las no transmisibles. En el caso de este último, son los primeros en detectar el aumento o disminución del consumo de sustancias estimulantes, la aparición de nuevos productos, nuevos tipos de adicciones, los cambios en los hábitos, el mecanismo de acceso a los productos.

Los agentes comunitarios de salud actúan de gestores en la promoción de la calidad de vida y salud en el territorio como puente entre el Servicio de Salud y la población, conociendo la realidad geográfica, social y sanitaria del territorio. Son de inestimable ayuda para la vigilancia epidemiológica. (Paraguay. Ministerio de Hacienda, 2011, p. 11).

La vigilancia es un bastión estratégico para la APS. Los ESF han logrado, además de la intensificación de la atención de la salud, una ampliación del radio de cobertura. La población accede a los servicios de salud y, en el mismo acto, el Estado accede al control de la misma. Con los ESF la presencia del Estado ha cobrado amplitud y profundidad. La cobertura de población atendida por las USF es significativa y favorece el acceso al derecho a la salud y a la superación de barreras geográficas, sociales, económicas y culturales, sobre todo para las poblaciones alejadas y con elevados niveles de pobreza, evidenciadas por el incremento de consultas y personas atendidas. (Paraguay. Ministerio de Hacienda, 2011, p. 11)

Una de las principales conclusiones, que expresa el documento de evaluación del Ministerio de Hacienda dice "Actualmente, se manifiesta un mayor involucramiento con la comunidad, buscan la atención integral, realizan un seguimiento de pacientes, se ve a toda la familia, sus problemas y sus condiciones de vida (Paraguay. Ministerio de Hacienda, 2011, p. 104).

Lo que surge, a primera vista, de la gran diferencia que existe a partir de la aplicación de la estrategia de APS es que ha aumentado enormemente el número de consultas (las personas que accedieron a los servicios de salud) y con ello, el registro institucional de la población ha aumentado ${ }^{7}$.

La política pública de salud, incluso si no logra mejorar los principales indicadores de la situación sanitaria, cumple un papel de primer orden en el sistema político. Jamás el Estado nacional ha penetrado tan profundamente en la vida de las personas, las familias y las organizaciones sociales como hasta ahora.

El Estado puede aprovechar las estructuras de los servicios de salud ${ }^{8}$, para cumplir objetivos políticos de toda índole a partir de la gestión de la información.

\section{Radiografía personal y social}

El censo es la confesión más amigable del sistema y donde el ACS organiza el cimiento de todo el edificio. "El censo comunitario es una práctica nueva muy importante para conocer el perfil de la población asignada y permite la interacción de las personas con los ESF" (Caballero, Denis, Flecha et al. 2010, p. 45). El objetivo del censo es establecer la relación confiable con las familias, el proceso no termina en la primera entrevista, ésta funda el punto de partida de una interacción. Luego está el aspecto de contenido: arrancar la información pertinentea través del conducto de la preocupación por la salud. Por esa

\footnotetext{
7 Personas atendidas por las USF, con las 704 USF se atendieron al $42 \%$ del total de personas atendidas en toda la red de servicios del MSPyBS en el año 2011 (Paraguay, Secretaría Técnica de Planificación, 2012, p. 11).

8 Desarrollo del sistema de informaciones: La APS recibe informaciones estadísticas provenientes de alrededor del 80\% de las USF, en un Informe mensual con formato estandarizado (Paraguay. Ministerio de Hacienda, 2011, p. 104).
} 
vía se puede preguntar todo, provocar la indagatoria es vital para el sistema político y hacerla pacífica y voluntariamente incorpora una eficacia técnica sin parangón con otra institución pública.

El censo de la USF (Paraguay, Ministerio de Salud Pública y Bienestar Social, 2011a) permite identificar las líneas fundamentales de información y las utilidades de las mismas para la estrategia biopolítica.

Además de la identidad personal, indaga bajo qué tipo de techo vive un individuo, cómo se moviliza, la extensión del núcleo familiar con el que convive. El censo permite acceder a la vinculación consanguínea y a la relación familiar nominal. El acto mismo de censar brinda acceso a informaciones que muestran los vínculos reales del entorno familiar con el individuo. Todas las observaciones deben estar escritas.

El censo es un documento de identificación personal y familiar. Es un prontuario de datos vitales y antecedentes socialesyeconómicos. El procedimiento hace posible introducir un factor fundamental en la relación individuo-Estado. El sistema utiliza la relación de confianza para acceder a las informaciones de las personas. Es inusual que una persona normal dé informaciones valiosas a agentes estatales con relativa facilidad. Habitualmente, las personas tienen recelo de los agentes de las instituciones públicas. Sin embargo, para los médicos, enfermeras o agentes comunitarios de salud las puertas están abiertas. Los protagonistas del censo instalan un evento que resulta una táctica poderosa para el acceso a intimidad de las familias.

La primera identificación es el documento de identidad. Si una persona no lo tiene, los miembros del ESF agencian o guían para proveerla. La recolección de la información y los mecanismos de investigación fundan la primera tarea interinstitucional. Ubicación geográfica, individualización precisa y registro son necesidades técnicas de la salud pública, de la policía y del gobierno. Si no tiene cédula de identidad civil, el agente del censo debe verificar si la persona posee certificado de nacimiento o certificado de nacido vivo, con los cuales se debe realizar el siguiente procedimiento identificatorio.

En el cuadro de los datos personales de la familia se consignan el nombre completo, la relación de parentesco con el jefe o jefa de la familia, la edad, fecha de nacimiento, sexo, nacionalidad, etnia, nivel educativo, su ocupación principal, las principales afecciones que padece y, si es mujer, si realiza o no planificación familiar. El cuidado y el control de la mujer son estratégicos, es la reproductora y también la principal agente de cuidados de salud en la familia y muchas veces en la comunidad.

En el cuadro de "principales afecciones" está incluido alcoholismo y tabaquismo, con lo que el ministerio de salud puede tener ubicada a la población afectada por las dos principales adicciones del país. Para el sistema sanitario es muy importante conocer la genealogía clínica del grupo familiar. Por eso en el interrogatorio se incluye "datos del fallecimiento de la familia”, la relación de parentesco, la edad de la persona fallecida, las causas y el tiempo en que ha ocurrido.

Con este procedimiento es posible determinar todas las razones, incluyendo las que no son enfermedades como accidentes, crímenes y suicidios. Las causas de mortalidad permiten tener un perfil de la familia, del territorio y de la zona. No es lo mismo una franja territorial afectada por muertes derivadas de problemas pulmonares que aquella cuya causa primera o segunda de muerte sea el asesinato o los accidentes de tránsito. El censo establece las coordenadas para conocer el perfil de los territorios violentos, de las zonas más traumáticas, de las condiciones sociales más vulnerables.

Para determinar la situación sanitaria es muy importante establecer un "perfil epidemiológico" de una región. Dicho instrumento refiere las afecciones 
más frecuentes de una población determinada. Una epidemia puede ser de enfermedades transmisibles como la fiebre amarilla, el paludismo o el dengue. Puede haber epidemias de enfermedades no transmisibles como la hipertensión, la diabetes, la obesidad. Existen otras epidemias que no son enfermedades y que afectan a la salud, como los accidentes de tránsito, los asesinatos, los suicidios. Otras epidemias están ligadas al modo de vida como el alcoholismo, el tabaquismo, la drogadicción.

Un perfil es una muestra de las características más o menos permanentes de una persona, una familia o un grupo social. Un perfil epidemiológico nos ilustra los sucesos más frecuentes de las enfermedades, hábitos o estilos de comportamiento o causas externas que afectan a la salud y la calidad de vida de las personas en una zona o región'.

Sin embargo, los perfiles, pueden no tener una utilidad sanitaria solamente. Una mirada policial o fiscal sobre estas informaciones recogidas permiten identificar otros diversos elementos y cruzarlos con los prontuarios policiales. Un archivo policial apenas tiene los antecedentes judiciales y civiles de una persona. Con el cúmulo de informaciones del estado de salud, de las condiciones de vida y de trabajo que le pueden proporcionar el ministerio de salud, la institución policial puede saber hasta la medicación cotidiana de una persona, sus hábitos, el comportamiento habitual en el territorio, sin hacer una sola pregunta. Es cierto, existen restricciones legales, que las produce el mismo Estado. Aquí lo importante es saber que el aparato estatal posee dichas informaciones y que tiene el poder de utilizarlos legal o ilegalmente.

El censo de los ESF indaga y observa

9 El perfil epidemiológico es la expresión de la carga de enfermedad (estado de salud) que sufre la población, y cuya descripción requiere de la identificación de las características que la definen. Entre estas características están la mortalidad, la morbilidad y la calidad de vida (Whittembury, sf, p. 1). sobre las condiciones de la vivienda, desde la propiedad, forma de pago o modo de ocupación, pasando por el tipo de material del techo, las paredes y los pisos, hasta la cantidad de habitaciones y el uso de las dependencias. Particular interés tiene la cantidad de dormitorios ya que de ella se deduce el grado de hacinamiento. Los servicios sanitarios de una casa indican aspectos de la calidad de vida de la familia y el status o nivel social.

El formulario registra información sobre el acceso al agua potable, la fuente y la forma de abastecimiento; si procede de una cañería fuera de la vivienda y dentro del terreno; si la cañería está dentro de la vivienda; si es de un grifo público o si se provee del vecino, del aguatero $u$ otros medios. El tipo de abastecimiento habla también del status.

El tratamiento de la basura dice muchas cosas, el censo da a conocer cuántas familias tiran sus desperdicios en los cursos de agua, en los baldíos, zanjas, chacras o en las calles y quienes están suscritos a sistemas de recolección privados o públicos.

El informe sanitario sobre una familia culmina con el tipo de desagüe y de baño que utiliza. Existen otras informaciones que se adquieren por el censo: los servicios de electricidad, teléfono, celular, los electrodomésticos con que se cuenta, los medios de transporte. Dónde acuden las personas cuando requieren atención a la salud, en este caso no se registra solamente si el servicio de salud es público o privado, o si tiene seguro médico, sino incluso, si la persona acude a un curandero. Es un dato adicional importante si existen embarazadas en la familia.

También es un dato de gran relevancia política si los miembros del grupo familiar participan en organizaciones comunitarias, permite identificar liderazgos, tipos de liderazgos, tendencias, afinidad de las familias hacia determinados problemas o preocupaciones. Una simple pregunta sobre la pertenencia a algún grupo o movimiento social que realizan 1800 ESF con un promedio de 3.500 personas por 
equipo, sirve para tener un mapa políticoideológico más allá del perfil de salud.

La superioridad del control territorial que realiza el MSPyBS se destaca en la meticulosidad del censo y, sobre todo, en la frecuencia del chequeo de la información.

Se llevan una serie de formularios de registro en las cuales se van detallando tanto las actividades de prevención como de promoción que se realizan. Se realizan visitas domiciliarias tanto por oferta (cuando el equipo decide visitar una comunidad determinada), 1-2 veces por semana; como por demanda (a requerimiento de individuos $o$ familias que reportan de algún familiar o conocido que está enfermo y que no puede asistir a las instalaciones sanitarias). (Paraguay. Ministerio de Hacienda, 2011, p. 2).

Las informaciones logradas en las visitas domiciliarias quedan registradas en documentos pre elaborados que deben reportarse mensualmente a la Región Sanitaria correspondiente y desde allí procesados y enviados al equipo central de la Dirección General de Información Estratégica en Salud (DIGIES) y a la Dirección General de Atención Primaria de Salud. (Paraguay. Ministerio de Hacienda, 2011, p. 32)

Cuando se establezcan todas las USF previstas (1800 unidades), todos los habitantes del país deberían estar censados y monitoreados mensualmente y las informaciones proveídas por los ESF dispuestos en una base de datos centralizado e informatizado. Aunque no sea así -porque los sectores de mayores recursos económicos no acuden a los servicios públicos o no accedan a dar información sobre sus vidas- el acceso a los sectores populares, trabajadores, campesinos, pobladores de los barrios y compañías está garantizado.

\section{Participación social y vigilancia \\ El control social cierra el esquema}

con la "participación comunitaria”. Nunca, tanta gente ha participado de manera voluntaria, entusiasta y comprometida en el funcionamiento del sistema de monitoreo institucional. Por vez primera el sistema de investigación tiene unos lazos en la población de manera estable. Los agentes sociales participan en forma voluntaria, entusiasta y comprometida.

La Atención Primaria de la Salud funciona como un mecanismo rizomático donde los elementos propiamente sanitarios se confunden con los políticos, donde las raicillas se entrelazan tan tupidamente que hace del control una forma de ser, una forma de actuar en favor de la comunidad. El aspecto sanitario hace al político. Adoptar un compromiso con la salud es apoyar la estrategia del Estado.

La participación es una técnica pastoral para que la población coopere con el sistema. Borra los límites entre la acción de la burocracia estatal y la de la comunidad (Estigarribia, 2014). Los mecanismos de prevención tales como los controles regulares, las inmunizaciones, las consultas periódicas producen un efecto de sociedad altamente medicalizada, consumidora de insumos y medicamentos $\mathrm{y}$, sobre todo, ligada a los sistemas de monitoreo del Estado.

Las campañas y mingas promovidas desde el poder político inculcan la participación de la población en la detección de los focos de contagio, ya sea en ambientes públicos o privados. De esta manera, permite que los individuos investiguen de diversas maneras $y$ detecten para el Estado las familias que no se sometieron a los procedimientos establecidos. Se instala, el mecanismo de vigilancia vecinal cuyas competencias aumentan en la medida en que aumenta el compromiso con la salud: detección de niños sin vacunar, violencia doméstica, adicciones. La medicalización permite crear hábitos sociales ligados al mejoramiento de la calidad de vida; permite crear hábitos de colaboración con las autoridades. Cuanto 
mayor sea el compromiso de los miembros de la sociedad con la salud, más eficaz se vuelve el sistema de control. Los modelos de comportamiento inculcados producen una diseminación de la moral del control, de la solidaridad como cogestión con el Estado.

La reunión es una de las fuentes de información más dinámicas porque permite hacer pronósticos e hipótesis sobre los comportamientos grupales. Los demás instrumentos de recolección de la información, como el censo o la ficha clínica, acentúan, completan, refuerzan y confirman los datos obtenidos.

La cooptación de la población es fundamental, porque implica unas imbricaciones que hacen posible el funcionamiento de todo el sistema aun sin médicos ni enfermeras. La vigilancia epidemiológica y la promoción de la calidad de vida dependen más del grado de confianza de la población en los agentes de salud que hacen la visita domiciliaria, el censo y la territorialización que la labor propiamente médica.

Las organizaciones sociales cambian de orientación. En vez de grupos reivindicativos, se convierten en organizaciones que tienen por actividades centrales la gestión de las asignaciones presupuestarias, la verificación de la ejecución, el monitoreo de la calidad del servicio y el cumplimiento de las metas establecidas por la autoridad sanitaria. La estrategia de APS involucra la acción institucional directa y la acción de los individuos y organizaciones sociales. La relación produce un compromiso político en nombre del derecho a la salud de las personas.

El Ministerio de Salud Pública promueve la participación de la ciudadanía a través de 3 procesos bastante diferenciados: a nivel de los consejos locales. A través de la participación como usuarios de los servicios. En acciones preventivas en salud (Paraguay. Ministerio de Hacienda, 2011).

En el caso de los consejos locales de salud, el $73 \%$ de los municipios los tiene y reciben fondos del MSPB. En el caso de la organización de usuarios, el MSPBS ha logrado crear grupos de hipertensos, diabéticos y embarazadas. (Paraguay. Ministerio de Hacienda, 2011). En las acciones preventivas, la evaluación reconoce la participación de la comunidad en las mingas ambientales durante la epidemia del dengue, en la realización del censo, en jornadas de sensibilización, en visitas domiciliarias, en procesos educativos, sumándose a las campañas de vacunación (Paraguay. Ministerio de Hacienda, 2011).

El documento de evaluación no menciona un aspecto que tiene mucha importancia en la estrategia de la APS en el Paraguay, la asamblea comunitaria o "Aty Guasu”. Éste y demás instancias de reunión y participación no son sino parte integrante extra oficial de los servicios, agentes de captación, instrumentos forjadores de consenso, ojos y oídos del sistema.

He aquí el análisis de la Guía del Aty Guasu, el documento marco de la participación comunitaria según la Dirección General de la Atención Primaria de Salud (DGAPS) del MSPBS.

a) Diagnóstico de los problemas y perfiles de solución: "En el marco de la APS la participación es la posibilidad de intervenir en la descripción de la problemática y el diseño de las respuestas a las necesidades" (Paraguay. Ministerio de Salud Pública y Bienestar Social, 2012b, p. 4).

b) Respuesta integral y participación activa: "El Ministerio de Salud inicia un proceso de transformación de la atención sanitaria para dar respuesta integral a las necesidades de las personas con la participación protagónica delas mismas" (Paraguay. Ministerio de Salud Pública y Bienestar Social, 2012b, p. 4).

Se han tomado dos ejemplos básicos que ayudan a ver el involucramiento de la población, o sectores de ella, en el plan 
de participación social del MSPBS en la campaña de vacunación y en la campaña contra la epidemia del dengue. En ambos casos, los participantes, unavez convencidos de la importancia de ambas campañas, apoyados con una abundante publicidad de los medios de comunicación masivos, proponen tácticas, sugieren intervenciones, ayudan a la identificación de las debilidades del proceso, indagan sobre los sectores no cubiertos por la campaña, detectan familias no vacunadas y viviendas descuidadas, cuyos patios están en desorden y pueden ser criaderos de vectores. "La participación social protagónica implica que las personas opinen, actúen y controlen las acciones que afectan a su comunidad para mejorar su calidad de vida y la de sus familias" (Paraguay. Ministerio de Salud Pública y Bienestar Social, 2012b, p. 14).

La línea de intervención es multiplicar la confianza en los equipos de salud, de servir de nexo para la detección de individuos y situaciones sociales medicalizables, de controlar el funcionamiento de las instituciones y por esta vía controlar a los controladores (Foucault, 2002), de dirigir la atención hacia aspectos hasta ahora no divisados.

El Aty Guasu "es una reunión local de ciudadanos y ciudadanas que tratan asuntos de interés de la comunidad" (Paraguay. Ministerio de Salud Pública y Bienestar Social, 2012b, p. 18). Cualquiera puede integrar el espacio, no hay formalidades, no hay quorum exigido, se puede considerar Aty con un número mínimo de asistentes. Y esto habla claramente de su carácter funcional al aparato institucional.

"Los Aty Guazú son espacios públicos abiertos donde los protagonistas son los habitantes de los territorios sociales. Permiten encontrarse, compartir saberes, valorar y respetar al semejante y diferente, construir consensos y respetar disenso" (Paraguay. Ministerio de Salud Pública y Bienestar Social, 2012b, p. 18). Es muy importante para el sistema que la gente se reúna $y$, por ello mismo, adquiere singular significación el hecho de que los participantes se sientan a gusto. Es una escuela de participación funcional. El consenso es el que articula el mecanismo de funcionamiento.

Más importante que las acciones que adopten es el hecho mismo de reunirse y conversar. El mecanismo de decisión es muy importante en los sindicatos, en los partidos políticos, en centros de estudiantes que se reúnen en asamblea y ésta debe terminar en resoluciones. Los Aty Guasu tienen rasgos diferentes, más laxos y difusos:

pueden ser realizados en diferentes escalas, donde los niveles más pequeños serían por cuadras, barrios o micro territorios; estableciendo encuentros entre los vecinos más cercanosdondetratartemasdeinterés, socializar información, plantear acciones o simplemente compartir y celebrar momentos significativos con los vecinos (Paraguay. Ministerio de Salud Pública y Bienestar Social, 2012b, p. 20).

La reglamentación no proviene de un estatuto autónomo sino del manual institucional. La agenda normalmente lo establece el Equipo de Salud de la Familia. Casi todos los problemas laborales, sociales, ambientales influyen en la salud y pueden ser discutidos como determinantes de la salud y por esa vía reducir los problemas más generales a un asunto comunitario, de gestión comunitaria.

Las acciones que brotan de estas instancias apuntan a la cooperación con el poder político y no a la acción de protesta o rechazo. Los ejes de discusión y acción de las instancias comunitarias son el involucramiento en la campaña contra el dengue, el apoyo a la campaña de vacunación, la detección de hipertensos y diabéticos, la atención a las familias que viven en condiciones infrahumanas, el seguimiento de las embarazadas. Las instancias de participación, promovidas desde el gobierno, son consideradas de 
“empoderamiento social” (Paraguay. Ministerio de Salud Pública y Bienestar Social, 2012b, p. 15) que fortalecen “... espacios de decisión comunitaria para el ejercicio de una democracia participativa" (Paraguay. Ministerio de Salud Pública y Bienestar Social, 2012b, p. 15).

El manual afirma: El Aty Guasu es para "Articular el trabajo entre los ciudadanos y las instituciones públicas, para implementar territorialmente políticas sociales que den respuestas a las necesidades de la gente" (Paraguay. Ministerio de Salud Pública y Bienestar Social, 2012b, p. 15). Las instancias de participación permiten extender el servicio sanitario, proceder a la atención continua, conllevan la medicalización de la vida social y de los problemas comunitarios.

Los principales trabajos premiados en la I Muestra Nacional de APS realizada en Asunción los días 10, 11 y 12 de mayo de 2012 (Paraguay. Ministerio de Salud Pública y Bienestar Social, 2011c) en el tema "actividades significativas", sobresalieron tres: un trabajo sobre la desmanicomialización, otro sobre violencia contra las mujeres y otro contra la desestigmatización de los consumidores de drogas. Todos son problemas sociales encarados como determinantes de la salud. Los ESF introducen finos hilos de detección, rodeo, acercamiento, abordaje y tratamiento de problemas difíciles de la sociedad; desmarginaliza a los individuos excluidos; sacan a la luz problemas de los cuales la gente no quiere hablar o habla sottovoce; involucra a miembros de la comunidad, a los familiares, para la asunción de las cargas de atención y tratamiento de los adictos, de la gente con problemas mentales y las víctimas de violencia doméstica. Aquí es importante el papel de la mujer como servicio ad honorem para el cuidado de adictos, pacientes encamados, niños, adultos mayores. El Estado saca bastante provecho de la situación opresiva de la mujer. Sobre ella caen todas las labores domésticas y también del cuidado. La mujer es un baluarte de la salud pública tanto para los servicios directos como para la formación de la subjetividad. Los niños interiorizan los roles, las creencias y valores promovidos por la madre, con la misma fluidez con que hablan la lengua materna. Ella es la encargada de garantizar que la familia esté dentro del sistema de salud, cumpliendo con los estándares establecidos.

La adicción a las drogas, la violencia doméstica, los problemas mentales son más fáciles de detectar cuando la investigación se realiza no desde una instancia represiva, cuando no es la policía o la fiscalía ni las autoridades del manicomio los que emprenden la tarea, sinodesdeinstituciones amigables. Los grupos comunitarios ligados a los ESF ganan confianza y, por esa vía, aumentan la capacidad de detección de los problemas del submundo, de lo más marginal, con calidad microscópica. Es, a un tiempo, la capacidad inquisitiva y la canalización de la política estatal a través de las organizaciones y líderes comunitarios.

\section{Conclusiones}

En el nombre de la ciencia y el derecho a la salud el poder político se otorga la tarea de cuidar la población y se instituye como administradora de la vida de los individuos. El Estado logra instaurar un sistema meticuloso y dinámico de observación de las personas y las organizaciones, a través del MSPBS. La estrategia de APS, a través de la cobertura preventiva y el registro permanente de los individuos y las familias, permea hasta las capas sociales más vulnerables para la inspección microscópica de los sectores populares. Los mecanismos apuntan a ejercer su dominio en un amplio abanico que involucre todos los aspectos de la salud, incluyendo los determinantes.

No es un mecanismo externo adherido a la política de salud, sino que es inherente al tratarse de una política de Estado, descansa en el carácter mismo del poder que irrumpe en el tejido social a través de la salud. 
La estrategia de APS es el más gigantesco sistema de información, investigación y control de la población que puede tener un Estado. En su plenitud de funcionamiento tendrá bajo observación a toda la población, incluyendo aquellos que aún no tienen identidad legal, no hayan nacido en instituciones sanitarias públicas o no asistan a instituciones educativas. No obstante, lo más importante no es tanto su magnitud sino su versatilidad, la flexibilidad en la relación con las personas, su capacidad de actualización.

Con la estrategia de la APS, toda la actividad individual y social está medicalizada. Es un tipo de gobierno pastoral que habilita a que nadie quede fuera del rebaño. La gestión de la salud y no de la enfermedad implica la medicalización de la vida con lo que se borra la distancia entre sanos y enfermos. No hay un tiempo en el cual el cuerpo puede estar fuera de la vigilancia sanitaria, todo el tiempo está biopolitizado. La gestión del cuerpo es tan importante como la subjetivación para la reproducción del orden social.

Los Agentes Comunitarios de Salud son fundamentales para la interacción entre las familias y las USF. Por medio de estos agentes, la vigilancia de la salud y de las condiciones de vida, se realiza con independencia de la acción y demanda de los pacientes. Su trabajo está en las casas de las familias, las escuelas, los lugares de trabajo, los lugares de culto, los espacios de encuentro, de esparcimiento y en las calles. Son los principales gestores de la confianza, consiguen información íntima que ningún otro funcionario del Estado puede obtener.

\section{Referencias Bibliográficas}

Bourdieu, P. (1998). Capital cultural, escuela y espacio social ( $2^{-}$ed.). México: Siglo XXI.

Caballero, M., Denis, V., Flecha, M. (2010). ProgramaEmblemático Gubernamental Unidades de Salud de la Familia. Informe de Evaluación de Proceso. Recuperado de http://www.mspbs. gov.py/planificacion/wp-content/ uploads/2012/o4/APS_Programa_ emblematico_Caballero2o10.pdf

Davis, S. L. \& Chapa, D. W. (2015). Feature: Social Determinants of Health: Knowledge to Effective Action for Change. The Journal For Nurse Practitioners, 11424-429. doi:10.1016/j. nurpra.2015.01.029

Deleuze, G. \& Guattari, F. (1994). Mil mesetas. Capitalismo y esquizofrenia ( $2^{\mathrm{a}}$ ed.). Valencia: Pre-textos.

Estigarribia, A. (2014). Políticas Públicas y control social. Asunción, Arandurâ.

Foucault, M. (1978). Medicina e Historia. El pensamiento de Michel Foucault. Washington, D.C.: Organización Panamericana de la Salud Oficina Sanitaria Panamericana, Oficina Regional de la OMS.

Foucault, M. (2002). Vigilar y castigar. El nacimiento de la prisión. Buenos Aires: Siglo XXI.

Foucault, M. (2006). Seguridad, territorio y población. Buenos Aires: Fondo de Cultura Económica.

Janowitz, M. (1975). Teoría y control social. Publicado en Delito y Sociedad. Revista de Ciencias Sociales, Año 4, $\mathrm{N}^{\circ} 6 / 7$, 1995. Recuperado de http:// www.catedras.fsoc.uba.ar/pegoraro/ Materiales/Janowitz_Teoria_Social_ Control_Social.PDF

Mackenbach, J. P. (2009). Politics is nothing but medicine at a larger scale: reflections on public health's biggest idea. Journal of Epidemiology and Community Health, 63(3), 181-184. doi:10.1136/jech.2008.077032

Martínez, V. (sf). Salud Pública, medicalización de la vida y educación. Recuperado de www.fedicaria. org / mi e mbros / fed A s turias / Apartado_7/SALUD\%2oPUBLICA\%20 M E DICA LIZACIO N\%2 O DE\% 20 LA\%20VIDA\%20Y\%2oEDUCACION_ VENANCIO.pdf

Oleskin, A. V. (2012). Biopolitics: The 
Political Potential of the Life Sciences. Hauppauge, N.Y.: Nova Science Publishers, Inc.

OMS (2009). Reducir las inequidades sanitarias actuando sobre los determinantes sociales de la salud. $62^{\underline{a}}$ Asamblea Mundial de la Salud. 22 de mayo de 2009. Recuperado de http:// apps.who.int/gb/ebwha/pdf_files/ A62/A62_R14-sp.pdf

OMS (2012). Determinantes sociales de la salud. Recuperado de http://www.who. int/social_determinants/es/

PAHO. Pan American Health Organization (1978). Carta de Alta Ata. Recuperado de http://www.paho.org/spanish/dd/ pin/alma-ata_declaracion.htm

PAHO. Pan American Health Organization (1986. Carta de Ottawa para la Promoción de la Salud. Recuperado de http://www.paho.org/Spanish/AD/ SDE/HS/OttawaCharterSp.pdf

Paraguay. Ministerio de Hacienda. (2011). Informe Final de Evaluación Programa de Unidades de Salud de la Familia. Periodo de evaluación Noviembre 2008-diciembre 2010. Asunción: Dirección General de Presupuesto. Departamento de Control de Ejecución y Evaluación Presupuestaria.

Paraguay. Ministerio de Salud Pública y Bienestar Social (2012b). Guía para el Aty Guasu comunitario. Asunción. MSPyBS.

Paraguay. Ministerio de Salud Pública y Bienestar Social (2012c). Guía de visitas domiciliarias. Asunción: MSPyBS.

Paraguay. Ministerio de Salud Pública y Bienestar Social (2012d). Manual de los/las agentes comunitarios/as de salud. Asunción: MSPyBS.

Paraguay. Ministerio de Salud Pública y Bienestar Social (2012e). Guía de territorialización. Asunción: MSPyBS.
Paraguay. Ministerio de Salud Pública y Bienestar Social. (2011). Hoja de censo, formulario № 1 . Asunción: Dirección General de Atención Primaria de Salud.

Paraguay. Ministerio de Salud Pública y Bienestar Social. (sf). ¿Qué es la Egi-Dengue? Recuperado de http://dengue.mspbs.gov.py/index. php?option=com.

Paraguay. Secretaría Técnica de Planificación (2012). Informe final de Monitoreo. Programa Atención Primaria de Salud, subprograma: APS medicina familiar del Ministerio de Salud Pública y Bienestar Social (borrador).

Rosen, G. (2005). De la policía médica a la medicina social ( $2^{\underline{a}}$ ed.). México: Fondo de Cultura Económica.

Segura del Pozo, J. (2009). Salud pública y biopolítica: la medicina social, según Virchow. Recuperado de http:// www.madrimasd.org/blogs/salud_ publica/2009/o1/o7/110752\#_ftnref5

Waitzkin, H. (2006). Un siglo y medio de olvidos y redescubrimientos: las perdurables contribuciones de Virchow a la medicina social. En Medicina social, Volumen 1, № 1 Junio 2006. Recuperado de http://www.medicinasocial.info/ index.php/medicinasocial/article/ view/14

Whittembury, A. (sf). El perfil epidemiológico y el análisis de situaciones del país. Lima. Recuperado de http://www.epiredperu.net/epired/ cursos/epidemiologia_bas-mh/ epibas_text16.pdf 